\title{
Numerical modeling of bumps using the stress stages
}

\author{
P. P. Prochazka \\ Association of Czech Concrete Engineers, Prague, Czech Republic
}

\begin{abstract}
In several of the author's previous papers the problem of bumps occurrence has been solved by distinct element methods, namely by free hexagon method. The latter method proved its significance in description of the most probable nucleation of cracks, which at the moment of bumps create the contact surface of the moving part of the coal seam. The movement of particles is also described in a very good manner. In this paper another approach is used. Starting with the aim of describing whether the bumps occur or not, or under which condition, the continuum of both rock seam and the overburden (rock) is considered. The decision whether the bumps are triggered can then be derived from conditions at different points of the coal seam. For the solution, finite element method is used and elastic behavior (more precisely brittle material) of the coal seam and plastic behavior of the rock are assumed.
\end{abstract}

Keywords: bumps in deep mines, mechanical behavior of rock, slip conditions.

\section{Introduction}

Bumps or rock bursts is a phenomenon, which occurs during mining in deep mines. This phenomenon appears during longwall mining, for example, and mostly it is qualified as a sudden release of energy concentrated at the side face of a shaft. This extreme concentration can be caused by material changes in the overburden rock massif, of distant local disturbances, due to creation of cracks, emission of gas, human activities in the neighborhood of the site of mining, etc. In any case, it is necessary not to forget the fact that along the upper part of the shaft where the ceiling and the rock are in contact, a tip of a crack (notch) causes a natural accumulation of energy. From classical theories based on elastic solution such a point is classified as a mass point of singularities and even 
involving plasticity in the neighborhood of this point the most danger threat of failure of the structure appears.

It is well known that fracture mechanics problems can be substituted by contact problems by introducing proper new parameters, which are connected with certain law along the contact. This law has to be selected very attentively. In most cases generalized Mohr-Coulomb hypothesis is adopted and the material parameters can be obtained from straightforward experiments. Such experiments are uneasy to carry out in the case of assessment of cracks. From these assumptions one discrete model is published, [1-2], and titled free hexagon method. Such a method seems to be very promising for solving bumps occurrence during longwall mining. The free hexagon method is similar to PFC method (particle flow code), which was established and applied in [3-4]. This approach consists in dynamical equilibrium of ball particles, substituting continuum of rock and coal seam. In [1-2] principal deviation from the PFC has to be introduced because of particular application on rock bursts in deep mines. Discrete element methods enable designers and researchers to describe the material behavior of structures from the point of view of damage mechanics, localization of stresses and fracture mechanics. Hexagonal shape of the elements is selected as the shape is "almost" circular (the shape of grains, stones, fibers, etc.) and at the same time it is possible to cover the domain describing the structure (or its parts) with the least geometrical error.

The application to geotechnical stability problems has very typical features. It is well-known that the mechanical behavior at the loss of stability of slopes, tunnel faces, retaining walls, etc., obeys an interesting phenomenon: localization of stresses along slip curves or surfaces, which causes the destruction (failure) of the rock and soil. There are certain methods starting with a formulation of the problem in the domain, describing the structure under consideration, which is a continuum. We can name "Cohesive zone method", [5], which deals with Barenblatt's theory, for example. In our problem of rock bursts such methods are on one side uneasy applicable and on the other side exhibit unreal behavior, according to a couple of test examples.

One of a possible experimental treatment was suggested in paper [6], where Araldit and similar physically equivalent materials are used. Based on models of such a similar conception coupled modeling was used in [7-9]. In [7] rock bursts are studied in mines at extreme depths. The free hexagon method is used to determine the bumps state in the rock. In [8] a similar problem is solved for more general cases occurring during mining. In [9] tunnel face stability is assessed.

In [10-12] results from on site measurements are published, recommendations are provided on how to proceed in determining the rock bursts and, what is the most important for restraining the rock bursts, and the way of movement is shown in these publications.

Both parts, the overburden and the coal seam (layer), are mechanically described by finite elements. For the sake of clearness the structure under consideration is depicted in Fig. 1. It is worth to note that the part of the rock considered into the numerical model is loaded from above in such a way that the 
mine possesses depth of $1000 \mathrm{~km}$. The part in the computation is considered as bearing, the above that one is only additional loading.

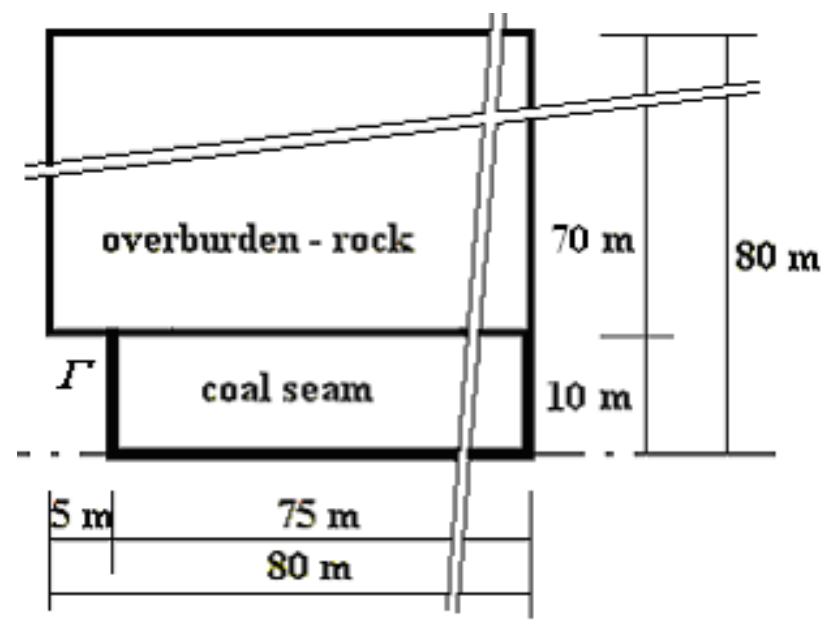

Figure 1: Vertical cut of the mine.

\section{Description of plasticity in the rock}

Before we tackle the plasticity definition, some basic formulas are operative to be mentioned in what follows. First, we start with elastic state. Since all the quantities involved in elasticity problem are connected with arbitrary coordinate system chosen and a priori fixed, of a great interest to us is to concentrate on what happens when we change the original coordinates $0 x y z$ and rotate them to a new system $0 x^{\prime} z^{\prime} y^{\prime}$ by an angle $\beta$. Since a sufficient condition to be fulfilled is to work in two-dimensional system, we can identify the $z$ '-coordinate with the $z$-coordinate of the original system.

The transformation of the symmetric stress tensor is subject to well-known tensor transformation:

$$
\sigma^{\prime}=\left[\begin{array}{ccc}
\sigma_{\mathrm{x}}^{\prime} & \tau_{x y}^{\prime} & \tau_{x z}^{\prime} \\
\tau_{x y}^{\prime} & \sigma_{y}^{\prime} & \tau_{y z}^{\prime} \\
\tau_{x z}^{\prime} & \tau_{y z}^{\prime} & \sigma_{z}^{\prime}
\end{array}\right]=\mathbf{T}\left[\begin{array}{ccc}
\sigma_{\mathrm{x}} & \tau_{x y} & \tau_{x z} \\
\tau_{x y} & \sigma_{y} & \tau_{y z} \\
\tau_{x z} & \tau_{y z} & \sigma_{z}
\end{array}\right] \mathbf{T}^{\mathrm{T}}
$$

where

$$
\mathbf{T}=\left[\begin{array}{ccc}
\cos \beta & \sin \beta & 0 \\
-\sin \beta & \cos \beta & 0 \\
0 & 0 & 1
\end{array}\right], \quad \mathbf{T}^{\mathrm{T}}=\left[\begin{array}{ccc}
\cos \beta & -\sin \beta & 0 \\
\sin \beta & \cos \beta & 0 \\
0 & 0 & 1
\end{array}\right]
$$


$\beta$ is an oriented angle of transformation (the angle between $x$ and $x^{\prime}$ ). Performing the multiplication of matrices yields the following formulas:

$$
\begin{gathered}
\tau_{x z}^{\prime}=\tau_{y z}^{\prime}=0, \quad \sigma_{z}^{\prime}=\sigma_{z}, \quad \sigma_{x}^{\prime}=\sigma_{x} \cos ^{2} \beta+\sigma_{y} \sin ^{2} \beta+\tau_{x y} \sin 2 \beta \\
\tau_{x y}^{\prime}=\frac{1}{2}\left(\sigma_{y}-\sigma_{x}\right) \sin 2 \beta+\tau_{x y} \cos 2 \beta
\end{gathered}
$$

From the condition of extreme normal stresses with respect to the angle of rotation $\beta$ principal stresses $\sigma_{1}, \sigma_{2}, \sigma_{3}$ are expressed as:

$$
\begin{gathered}
\sigma_{1}=\frac{\sigma_{x}+\sigma_{y}}{2}+\sqrt{\left(\sigma_{x}+\sigma_{y}\right)^{2}+4 \tau_{x y}^{2}}, \sigma_{2}=\frac{\sigma_{x}+\sigma_{y}}{2}-\sqrt{\left(\sigma_{x}+\sigma_{y}\right)^{2}+4 \tau_{x y}^{2}}, \\
\sigma_{3}=\sigma_{z}
\end{gathered}
$$

The relation of normal and shear stresses (depending on the angle $\beta$ ) and the principal stresses can be displayed by virtue of the Mohr-Coulomb circle drawn in the coordinate system $0 \sigma \tau$. The center of the circle is derived as $\frac{\sigma_{1}+\sigma_{2}}{2}$ and the radius as $\frac{\sigma_{1}-\sigma_{2}}{2}$. From the above considerations it is clear that the relation is defined at an arbitrary point of domain describing the body under study. Moreover, it can be shown that if the principal stresses change the envelope of the circle approximately creates a straight line for negative normal stress. If the tensile strength is different from zero, the course of the envelope continues to the domain $\sigma>0$ as a circular segment. The cut of the envelope as the point $\sigma=0$ is called cohesion in soft rock or soil, and is called shear strength in rocks. The angle of slope of the envelope is the angle of internal friction, denoted mostly as $\alpha$. In this way new material characteristics are entering the description of the material behavior in the rock overburden.

The plastic behavior of the rock is described in the following way, see Fig. 2.

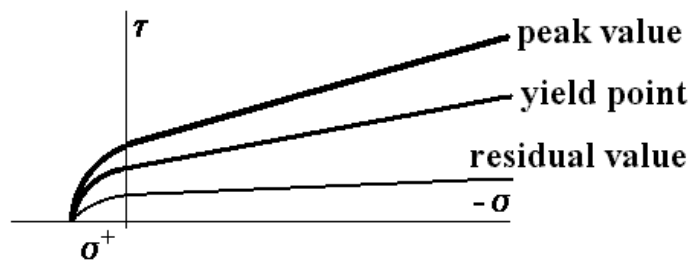

Figure 2: Description of plasticity stages.

If the point $(\tau, \sigma)$ is positioned below the envelope describing the yield point of the rock, elastic state is held. If this point belongs between the envelope "peak value" and "yield point", bilinear distribution of plasticity is considered. After 
reaching the "peak value" the drop to the "residual level" is required. The movement of the point $(\tau, \sigma)$ is done in such a way that orthogonal projections to the upper level envelopes are prescribed.

In order to describe the envelopes, two material characteristics are necessary to be determined from the experiments: the shear strength and the angel of internal friction both defined for each envelope.

From the above considerations it follows that irreversible process is described (due to the drop to the residual level). In soil mechanics this obstacle is overcome by increasing the volume weight. In our case we exploited Eshelby's forces applied along the boundary of the opening $\Gamma$, see Fig. 1. The forces increase in successive steps and the plasticity rule above described is employed.

Recall the meaning of Eshelby's forces. First the virgin state of both rock and coal is considered. On the boundary $\Gamma$ tractions are calculated from the stresses along this boundary (the mine is still filled in by the coal. As no mechanical nonlinearity is expected in the virgin state, the stress state in both rock and coal seam after excavating coal from the shaft is described by the calculated tractions being applied on $\Gamma$. Because zero tractions should act on $\Gamma$, the virgins state is superposed with the stress state due to the tractions on $\Gamma$. As for the displacements, they are calculated from the tractions only.

\section{Assessment of stability of the coal seam}

There is a plenty of numerical models dealing with description of bumps, see enclosed references. Few of them are concentrated on prediction of failure of the side face of the shaft. Starting from discrete element methods, continuum methods (cohesive zone) and finite or boundary elements the results are interesting, extending the nowadays knowledge, but for designers are not too much usable. A movement of particles can even be described. The last phenomenon is interesting, but has very small meaning considering decision on how the face would behave under critical condition. The most important result for consulting engineers and designers of strategy of mining is decision on whether or not the face fail, whether or not rock burst occurs.

The idea of determining the most probable bumps occurrence originates from parallel considerations pertinent with the field of large scale problems of stability of slopes. In classical theories (Peterson, Hultin, Felenius, Sarma, and others) a measure of stability is sought, which is called safety margin. We try to define such a safety margin, for which an array of its values will be created in the domain of coal seam and where the values are critical. We even, in the contrary to the slope stability problems, the course of disconnecting of the moving part and the stable part of the coal seam can be displayed.

The safety margin $k$ is defined as

$$
k \equiv k(\beta)=\frac{-\sigma(\beta) \tan \varphi+c}{\tau(\beta)}
$$

since for stable state an inequality has to be fulfilled: 


$$
\tau(\beta) \leq-\sigma(\beta) \tan \varphi+c
$$

where $\sigma$ and $\tau$ are normal and shear stresses, respectively, both depending on the angle $\beta ; \varphi$ and $c$ are respectively the angle of internal friction and the cohesion (in this case of hard rock we rather speak about shear strength).

Now select a point in the domain of coal seam. As the safety margin depends on the angle $\beta$, the minimum of $k$ in (4) is searched for. Since the material constants $\varphi$ and $c$ are given, the stresses are the only functions, which influence the value of the safety margin. Using the formulas (1) or (2) ( $\sigma$ substituting for $\sigma_{x}^{\prime}$ and $\tau$ for $\tau_{x y}^{\prime}$ ) the problem leads to seeking minimum of $k$, which we denote as critical safety margin $k_{\mathrm{cr}}$. This value is important for the point, the critical safety margin has been calculated at.

The same approach can be carried out at every point of the domain defining the coal seam and contour lines (hypsography) describe the level of danger of failure, of bumps.

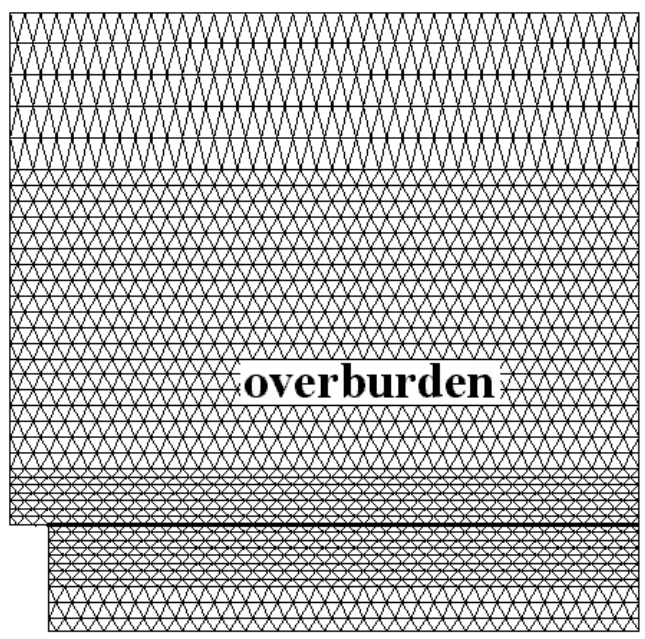

Figure 3: $\quad$ Meshing of the model.

\section{Example}

Couple of examples has been studied using the above described procedure. One of them is described here in more details. In the rock the following material properties has been used:

$E=21 \mathrm{GPa}, E_{\mathrm{p}}=15 \mathrm{GPa}, E_{\mathrm{r}}=2 \mathrm{GPa}, v=0.16, v_{\mathrm{p}}=v_{\mathrm{r}}=0.46, \sigma_{+}=5 \mathrm{MPa}$, $c=5 \mathrm{MPa}, c_{\mathrm{p}}=4 \mathrm{MPa}, c_{\mathrm{r}}=0 \mathrm{MPa}, \varphi=32^{\circ}, \varphi_{\mathrm{p}}=25^{\circ},, \varphi_{\mathrm{r}}=10^{\circ}$, and the plasticity is described as bilinear distribution with the vertex at the middle of the domain (i.e. $50 \%$ belong to the first straight line and $50 \%$ is the straight line in the second part). 
Similarly for coal we used such values of material characteristics: $E=7 \mathrm{GPa}, v=0.3, \mathrm{Nyp}=.3, \sigma_{+}=1 \mathrm{MPa}$, the strength is $38 \mathrm{MPa}$.

In the following pictures the geometry of the coal seam with the bearing part of overburden and triangular mesh of the finite elements (Fig. 3), principal stresses $\sigma_{1}$ (Fig. 4), $\sigma_{2}$ (Fig. 5), and principal $\tau$ (Fig. 6) are depicted for the fully elastic state for comparison with the real state. In Fig. 7, the hypsography of critical safety margin is illustrated, the most danger slip curve from which can be specified.

From Fig. 7 it obviously follows that this case is inclinable to bumps, as the safety margin is very low in a large area of the coal seam.

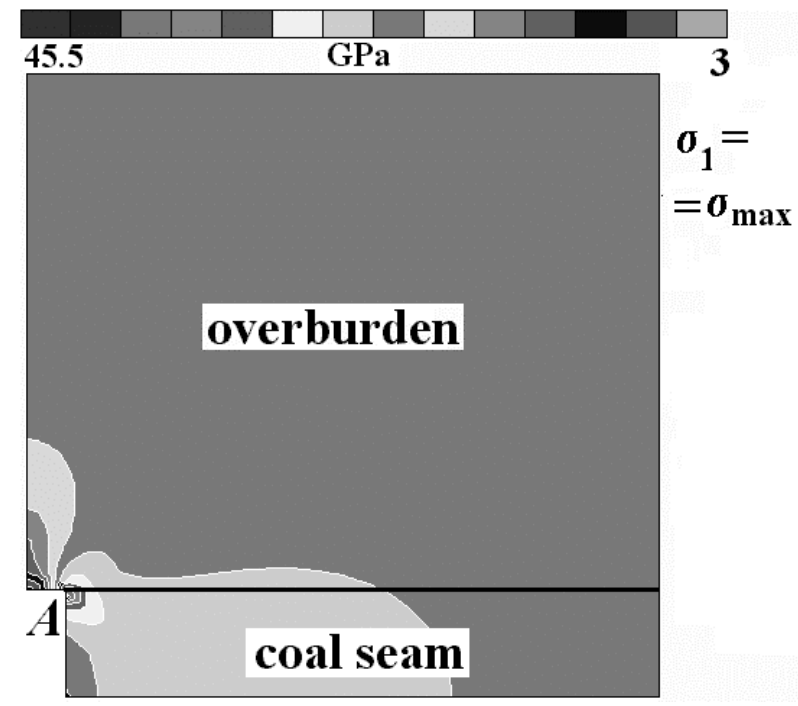

Figure 4: Hypsography of the first principal stress.

\section{Dynamical effects}

Consider first a particular element, which is along one boundary loaded by some force $P$. Because our model starts with realization of spring contacts, we can identify $P$ as a linearly dependent function of displacement $s$, i.e. $P=E s$, where $E$ is the spring stiffness (Young's modulus). The equilibrium of potential and kinetic energies is written as: $P s=1 / 2 m v^{2}$, where $m$ is the mass of the element and $v$ is the velocity. Connecting both last relations we easily get:

$v=\sqrt{2 \frac{P^{2}}{m E}}$, and $P$ is the normal force at which the extrusion occurs and is calculated from the numerical approach. The dynamics of the not extruding part of the seam is computed as initial problem with initial conditions: no displacement is at the initial time and the velocity is prescribed by the previous formula. The dynamics of the seam and the overburden can then be obtained, but 
from the practical goal it plays negligible role. The same is true for extruding part of the seam.

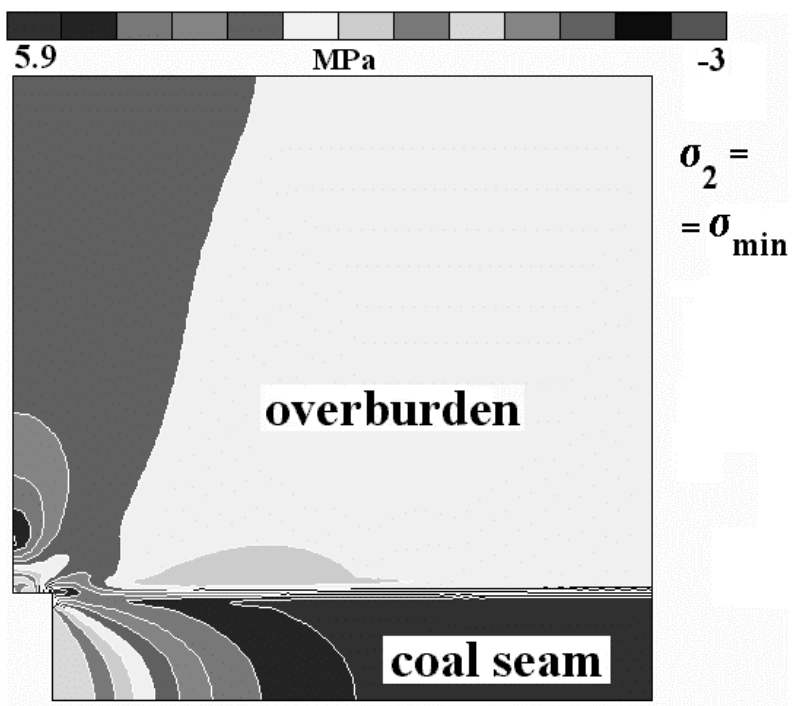

Figure 5: Hypsography of the second principal stress.

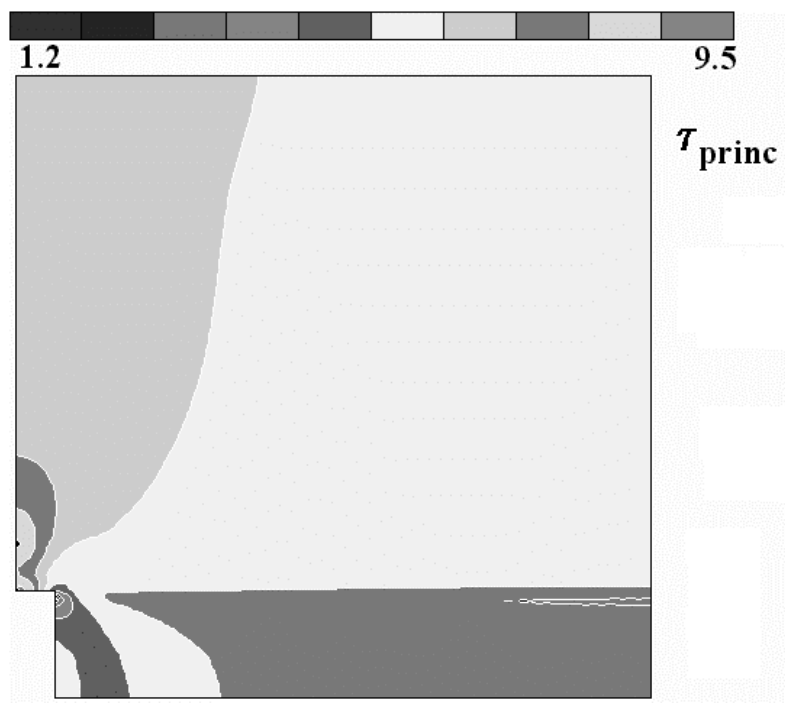

Figure 6: Hypsography of the shear principal stress.

\section{Conclusions}

In this paper quite new approach to design and assessment of bumps occurrence in deep mines is suggested. It starts with a special description of plastic material 
behavior and assessment of safety margin from high precision numerical methods. The suggested approach seems to be very promising for further research, where also hereditary problems should be involved, including the influence of the velocity of excavation of coal.

Since the plasticization process in the overburden is concentrated only to a close neighborhood of the shaft, the overburden for numerical computation is fully sufficient. As this process is irreversible, the Eshelby forces have to be introduced along the surface $\Gamma$ and they successively increase their values from zero to the value given from previous evaluation of virgin state in the rock and coal. It is worth noting that similar process has to be applied in the case of excavating ditch, although the plasticity rule admits the computation without successive loading of the Eshelby forces.

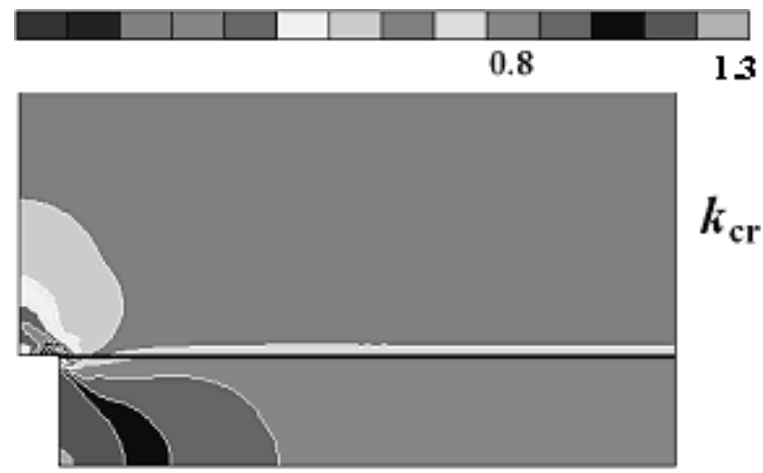

Figure 7: Distribution of critical safety margins and the most probable disconnection.

\section{Acknowledgment}

This research was supported by Grant Agency of the Czech Republic - grant number 103/05/0334.

\section{References}

[1] Procházka, P. Application of discrete element methods to fracture mechanics of rock bursts. Engng. Fract. Mech. 2003.

[2] Procházka, P. \& Válek, M. The BEM Formulation of Distinct Element Method. BETECH XXII. Cambridge: WIT Press, 2000, 395-404.

[3] Cundall, P.A. A computer model for simulation progressive large scale movements of blocky rock systems. Symposium of the international society of rock mechanics, 132-150, 1971.

[4] Moreau, J.J. Some numerical methods in multibody dynamics: Application to granular materials. Eur. J. Mech. Solids, 13, 4, 1994, 93114. 
[5] Elices, M., Guinea, G.V., Gomez, J. \& Planas, J. The cohesive zone model: advantages, limitations and chalenges. Engineering Fracture Mechanics 69, 2002, 137-163.

[6] Kuch, R., Lippmann, H. \& Zhang, J. Simulating coal mine bumps with model material. Rockbursts and seismicity in mines, Gibowitz \& Lasocki (eds.), Balkema, Rotterdam, 1997, 23-25.

[7] Vacek, J. \& Procházka, P. Behaviour of Brittle Rock in Extreme Depth. Our World in Concrete \& Structures. Singapore: CI-Premier, 19, 2000, 653-660.

[8] Vacek, J. \& Procházka, P. Rock Bumps Occurrence during Mining. Computational Methods and Experimental Measurements X. Southampton : WIT Press, 2001, 437-446.

[9] Procházka, P. \& Vacek, J. Comparative Study of Tunnel Face Stability. Damage \& Fracture Mechanics VII. Southampton : WIT Press, 2002, 163172.

[10] Haramy, K.Y. and Morgan, T.A.\& DeWaele, R.E. A method for estimating western coal strengths from point load tests on irregular bumps. 2nd Conf. on Ground Control in Mining, West Virginia University, July 19-21, 1982, 123-136.

[11] Haramy, K.Y., Magers, J.A. \& McDonnell, J.P. Mining under strong roof. 7th Int. Conf. on Ground Control in Mining, Bureau of Mines, Denver, USA, 1992, 179-194.

[12] Harami, K.Y. \& Brady, B.T. A methodology to determine in situ rock mass failure. Internal report of Bureau of Mines, Denver, CO, USA, 1995. 